\title{
Physical Activity and Glycemic Control Status in Chinese Patients with Type 2 Diabetes: A Secondary Analysis of a Randomized Controlled Trial
}

\author{
Wei-Yuan Yao ${ }^{1,+}$, Meng-Ge Han ${ }^{1, \dagger}$, Giuseppe De Vito ${ }^{2} \mathbb{1}$, Hong Fang ${ }^{3}$, Qinghua Xia ${ }^{4}$, Yingyao Chen ${ }^{5}$, \\ Xiaona Liu ${ }^{1}$, Yan Wei ${ }^{1,5}$, Russell L. Rothman ${ }^{6}$ and Wang-Hong Xu ${ }^{1,5, *(D)}$
}

1 Key Laboratory of Public Health Safety (Ministry of Health), School of Public Health, Fudan University, Shanghai 200032, China; 19211020004@fudan.edu.cn (W.-Y.Y.); 16211020051@fudan.edu.cn (M.-G.H.); 14211020005@fudan.edu.cn (X.L.); 14111020034@fudan.edu.cn (Y.W.)

2 Department of Biomedical Sciences, University of Padova, 35131 Padova, Italy; giuseppe.devito@unipd.it

3 Minhang District Center for Disease Control and Prevention, Shanghai 201102, China; mhcdcfh@126.com

4 Changning District Center for Disease Control and Prevention, Shanghai 200051, China; xiaqinghua56@126.com

5 Key Laboratory of Health Technology Assessment (National Health Commission), School of Public Health, Fudan University, Shanghai 200032, China; yychen@shmu.edu.cn

6 Department of Medicine, Vanderbilt University Medical Center, Nashville, TN 37232, USA; russell.rothman@vanderbilt.edu

* Correspondence: wanghong.xu@fudan.edu.cn; Tel.: +86-21-54237679

check for

updates

Citation: Yao, W.-Y.; Han, M.-G.; De Vito, G.; Fang, H.; Xia, Q.; Chen, Y.; Liu, X.; Wei, Y.; Rothman, R.L.; Xu, W.-H. Physical Activity and Glycemic Control Status in Chinese Patients with Type 2 Diabetes: A Secondary Analysis of a Randomized Controlled Trial. Int. J. Environ. Res. Public Health 2021, 18, 4292. https://doi.org/ 10.3390/ijerph18084292

Academic Editor: Paul Tchounwou

Received: 26 February 2021

Accepted: 9 April 2021

Published: 18 April 2021

Publisher's Note: MDPI stays neutral with regard to jurisdictional claims in published maps and institutional affiliations.

Copyright: (c) 2021 by the authors. Licensee MDPI, Basel, Switzerland. This article is an open access article distributed under the terms and conditions of the Creative Commons Attribution (CC BY) license (https:// creativecommons.org/licenses/by/ $4.0 /)$.

\begin{abstract}
This secondary analysis was designed to evaluate the independent effect of physical activity (PA) on hemoglobin A1c (HbA1c) level in Chinese patients with type 2 diabetes mellitus (T2DM). A total of 799 T2DM patients from eight communities of Shanghai, China, were randomized into one control arm and three intervention arms receiving 1-year interventions of health literacy, exercise, or both. PA was measured using the International Physical Activity Questionnaire at baseline, 12 months, and 24 months and quantified as metabolic equivalents (Mets). A multiple level mixed regression model was applied to evaluate the associations between PA and $\mathrm{HbA1c}$ level. After adjusting for potential confounders including interaction of PA level with initial PA or HbA1c, a significant improved $\mathrm{HbA} 1 \mathrm{c}$ was observed for the patients in the medium versus the lowest tertile groups of overall PA at 12 months $(\beta$ : $-3.47,95 \% \mathrm{CI}:-5.33,-1.60)$ and for those in the highest versus the lowest tertile group at 24 months $(\beta$ : $-0.50,95 \% \mathrm{CI}:-1.00,-0.01)$, resulting in a $\beta(95 \% \mathrm{CI})$ of -3.49 (95\% CI: $-5.87,-1.11)$ during the whole two-year period of follow-up. The negative association was also observed when the subjects were classified according to their exercise levels using the World Health Organization (WHO) recommendation as a cut-off point. The beneficial effect of higher PA level was only observed among patients having a lower level of baseline HbA1c or PA or both (all $p$ values for interaction $<0.05$ ). Our results provide evidence for the beneficial effect of PA and suggest that the exercise intervention should be addressed to the physically inactive patients to improve their PA level to a physiological threshold.
\end{abstract}

Keywords: randomized control trial; type 2 diabetes; physical activity; glycemic control

\section{Introduction}

Type 2 diabetes mellitus (T2DM) is one of the most common non-communicable diseases around the world [1]. Self-management behaviors such as diet control, physical activity (PA), medical adherence, and self-glucose monitoring have been suggested to achieve better glycemic control status and clinic outcomes and thus reduce substantial physical, psychological, and socioeconomic burdens of the disease at both family and society levels $[2,3]$. 
PA was defined as any body movement produced by skeletal muscles that results in energy expenditure [4]. The healthy and functional benefits of being active for T2DM patients have been well recognized, although the value of diet and exercise was not that helpful in the LOOK Ahead Trial [5]. Regular PA helps to maintain healthy weight and metabolic balance, improves cardiorespiratory fitness, lipid profiles, and musculoskeletal health, and thus reduces incidence of complications and all-cause mortality in T2DM patients [6,7]. In addition to leisure time physical activities, occupational, commuting, and domestic activities were also equally beneficial for human health $[8,9]$.

Possibly due to its delayed health effect, the need for persistent commitment, sweating, unpleasant feelings, and even pain [10,11], regular exercise is one of the most difficult self-management behaviors to follow for T2DM patients. Adults with diabetes are recommended to engage in at least $150 \mathrm{~min}$ of moderate to vigorous-intensity exercise per week $[12,13]$. However, epidemiological studies suggest that most patients were insufficiently active, with only $23-37 \%$ patients reaching the recommended exercise level in the USA, 21\% in Canada, and 15.3\% in mainland China [14-16]. Numerous strategies have been proposed to increase the adoption and maintenance of regular PA in T2DM patients. However, there is still a big gap in identification of the best PA intervention that can maximize glucose control and be sustained over the long term [17]. In recent years, a novel intervention was designed focusing on the health literacy (HL) of diabetes patients, namely, on patients' abilities to obtain, understand, and communicate basic information needed to make appropriate health-related decisions [18]. The HL-oriented intervention has been observed to improve glycemic control status effectively by enhancing self-management behaviors $[19,20]$.

In a cluster randomized controlled trial (RCT) conducted in Shanghai, China, we found that both HL and exercise-focused interventions could improve hemoglobin A1c (HbA1c) level in Chinese patients with T2DM [21]. In this study, the PA level of the patients, not only for leisure-time activities but also for commuting and domestic activities, could be improved by the exercise-focused intervention and the exercise module of the PRIDE toolkit used in the HL intervention (Supplementary Figure S1). Therefore, the independent effect of PA level on glycemic control remained unclear.

To evaluate the improved PA levels by the interventions, and the effect of PA on improvements in $\mathrm{HbA} 1 \mathrm{c}$, we conducted a secondary analysis of the data derived from the trial conducted in Chinese T2DM patients.

\section{Materials and Methods}

\subsection{Study Design and Subjects}

This study was a secondary analysis of a cluster RCT registered with the International Standard Randomized Controlled Trial (Trial registration: ISRCTN76130594; registration date, 12 January 2015). The trial was designed to evaluate the effectiveness of a comprehensive HL strategy, an exercise-focused intervention, or a combination of both on glycemic control and other outcomes in Chinese T2DM patients. A full description of the trial, including the methods for participant recruitment, has been published previously [22]. Briefly, eight Community Healthcare Centers ( $\mathrm{CHCs}$ ) were selected by convenience from a total of $26 \mathrm{CHCs}$, with 4 from Minhang District and 4 from Changning District of Shanghai, China. From each CHC, 3 to 5 clinic sites were selected. All clinical sites meeting the following criteria were selected from each center: (i) at least 20 patients can be recruited per site; (ii) a general practitioner (GP) team including at least 2 to 4 physician(s), nurse practitioner(s), or diabetes educator(s) per site can participate in the intervention; (iii) agree to participate for a minimum of 2 years; and (iv) agree to be randomized to any arm of the study.

A total of 799 diabetes patients were recruited from the 35 selected clinic sites during the period of February 2015 and March 2016, as described in our previous report [21]. The inclusion criteria for participants included clinical diagnosis of T2DM, 18 to 85 years of age, most recent $\mathrm{HbA} 1 \mathrm{c} \geq 7.5 \%$ and/or fasting glucose level $\geq 10 \mathrm{mmol} / \mathrm{L}$, and willing to 
participate in the project for the full 2-year duration. Patients were excluded if they had poor visual acuity (vision worse than $0.1 / 4.0$ using the Standard Logarithmic Visual Acuity Chart), significant dementia or psychosis (by health provider report or chart review), or terminal illness with anticipated life expectancy less than 2 years.

Randomization occurred at the level of the Community Healthcare Centers. Six Centers (35 clinic sites) were randomized to receive interventions, with two centers (9 clinic sites) focusing on HL-oriented intervention, two (9 clinic sites) focusing on exercise intervention, and two (9 clinic sites) focusing on comprehensive interventions, while two centers (8 clinic sites) were randomized into the control condition.

Ethics approval was obtained from the Medical Ethics Committee of Fudan University (IRB00002408 \& FWA00002399) before recruiting study participants (registration number: 2013-06-0451). All local medical ethics committees agreed with this approval according to the ethical standards of the Declaration of Helsinki, and all participants provided written informed consent. This study followed the CONSORT guidelines for reporting [23].

\subsection{Interventions}

The control group received usual diabetes care according to the Chinese National Guidelines [24], which includes conventional clinical consultations and treatment provision according to existing knowledge and at the individual clinician's discretion.

Patients in the exercise group received usual diabetes care and were asked to walk 3-5 times a week, 30-40 min per time in the first 6 months and 60-70 min per time in the following 6 months. They could walk continuously or divide the exercise into shorter time periods but for at least $10 \mathrm{~min}$ at a time. The intensity of exercise was ideally kept between 12 and 15 in the Borg RPE (Rating of Perceived Exertion) visual scale [25]. In addition, the patients in the group were required and trained by the GPs to record the frequency, time, and intensity (Borg scores) of walking in calendar books specially designed for the trial during the one-year period of interventions for the purpose of supervision.

In addition to usual diabetes care, the intervention for the HL group included use of a Chinese adapted version of the Partnership to Improve Diabetes Education (PRIDE) toolkit to aid healthcare provider-patient communications about diabetes management and a Clear Health Communication Curriculum for healthcare providers to improve diabetesrelated counseling communication skills, with specific attention to issues of literacy and numeracy. The PRIDE toolkit has 24 educational modules covering all components of diabetes self-management including diet, exercise, foot care, glucose monitoring, medication management, etc., which was written at a low literacy level with many pictures, white space, and other accommodations for low literacy patients [26,27]. The exercise module includes information on benefits of being active, fun ways to be more active, the dos and don'ts in exercise, as well as goal-setting tasks (Supplementary Figure S1). The Chinese version of the materials was used by health care providers in the HL group during regular patient-related visits at least 3 times a month, 5 to $7 \mathrm{~min}$ per time. At each visit, the providers were asked to cover at least two components from the toolkit materials as well as to perform and document at least one goal-setting task with the patient.

Before initiation of the HL intervention, providers gathered to obtain an approximately 5 to 6-h training on diabetes management, introduction of the Chinese version of the PRIDE toolkit, clear health communication skills, and application of the toolkit using principles of clear health communication. A post-training certification process was performed to ensure that providers had known how to use the materials.

Patients in the comprehensive intervention group received usual diabetes care and both HL and exercise interventions.

\subsection{Data Collection and Assessment of PA Level}

Information on demographic characteristics, diagnosis of diabetes, lifestyle factors including dietary habits (measured by 3-day 24-h dietary recall), and anti-diabetes agent use was collected at baseline through in-person interviews. All subjects were followed up 
at the end of 1-year interventions and 1-year post-interventions (Figure 1). HbA1c level was measured at baseline and at each follow-up survey using point-of-care high-performance liquid chromatography available in each Community Healthcare Center.

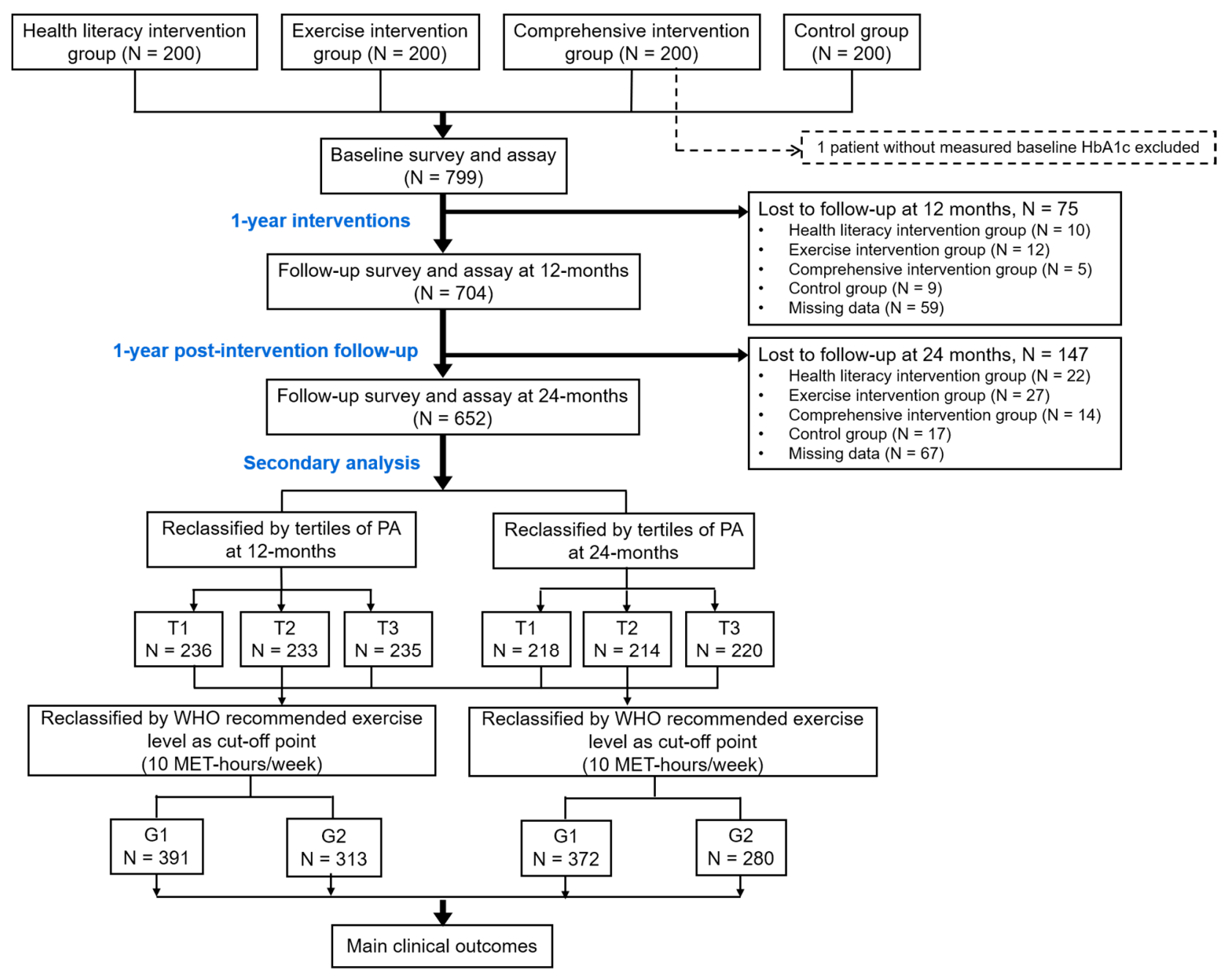

Figure 1. Flow diagram of the study. PA: Physical Activity; T1-T3: Group 1-3 by tertiles of PA; G1-G2: Group 1-2 by WHO (World Health Organization) recommended exercise level.

Considering that both HL and exercise-focused interventions were designed to improve PA level by integrating the activities into daily life, we assessed the PA level using the validated International Physical Activity Questionnaire (IPAQ) [28] instead of a walking specific IPAQ [29] at baseline as well as 12- and 24-month follow-up. Leisure-time activities included moderate- and vigorous-intensity exercises, which were defined as activities that cause a small increase in breathing or heart rate such as dancing, yoga, or tai chi, and exercises causing large increases in breathing or heart rate such as running, playing basketball, boxing, etc., respectively. Commuting activities included bicycling and walking during commuting, exercise, or shopping that last at least $10 \mathrm{~min}$. Domestic activities included several key household tasks such as cooking or preparing food, washing dishes, doing laundry, cleaning the house, and child care that last at least $10 \mathrm{~min}$ each time. Sedentary time included time spent on TV watching, computer using, video game playing, and reading at home, in the car, or with friends, but not during working. Participants were asked about the frequency and average time spent for each type of PA in a typical week.

The PA level was quantified as metabolic equivalent (MET)-hours/week by multiplying the METs, duration, and frequency of activities from exercise, transportation and 
housework [30,31]. The value of METs for each PA was recommended by the World Health Organization (WHO) guideline: 4 METs for moderate exercise, 8 METs for vigorous exercise, 4 METs for both cycling and walking, and an average value of 3 METs was assigned to calculate the energy expenditure in domestic activities according to the Compendium of Physical Activities [30,32].

\subsection{Reclassification of Subjects by Changes in PA Level}

All subjects were classified into three groups by the tertile of overall PA levels at 12 months and at 24 months, respectively. We also reclassified the participants into two groups using the WHO recommended level for exercise (10 METs-hours/week or 600 METsmins/week) as the cut-off point [32].

\subsection{Statistical Methods}

Continuous variables were presented as mean and standard deviation (SD) or medians and interquartile range (IQR), while categorical variables were presented as frequency and percentages. Comparisons of baseline characteristics by intervention status and PA groups were conducted using one-way analysis of variance (ANOVA) or Kruskal-Wallis tests for continuous variables and chi-square tests for categorical variables. Multiple-level mixed regression models were applied to estimate the associations of PA level with $\mathrm{HbA1c}$ after adjusting for age, sex, duration of diabetes, anti-diabetes drug or insulin use, monthly income level, initial levels of $\mathrm{HbA} 1 \mathrm{c}$ and $\mathrm{PA}$, and two-way or three-way interactions of PA with initial levels of PA and HbA1c. Since no significant difference was observed in characteristics between participants and those lost to follow-up, all missing values (95 at 12 months and 147 at 24 months) were excluded from the analyses. Moreover, we did not observe a significant heterogeneity in associations between PA and HbA1c by sex; we conducted analyses in all subjects.

All analyses were conducted using SAS 9.4 (SAS institute, Inc., Cary, NC, USA). Two-sided $p$-values $<0.05$ were considered statistically significant.

\section{Results}

Of a total of 799 study participants, complete data were available for 704 patients $(88.1 \%)$ at the end of intervention (12 months) and 652 patients $(81.6 \%)$ at 1-year postintervention (24 months). Supplementary Table S1 shows the baseline characteristics of all participants by intervention status. No significant difference was observed across the four groups on age, sex, tobacco and alcohol use, duration of diabetes, and $\mathrm{HbA} 1 \mathrm{c}$ level. However, the four groups were not comparable on education, HL and numeracy levels, monthly income per capita, and use of anti-diabetes agents.

Regarding the PA level, the overall and specific type of PA levels were significantly higher in the control arm than in the three intervention groups, as shown in Table 1. Further analysis showed higher baseline levels of PA in participants with lower $\mathrm{HbA} 1 \mathrm{c}$ levels regardless of PA type, but the differences reached significant only for commuting activities in women (Supplementary Table S2).

The significant effects of $\mathrm{HL}$ and exercise interventions on $\mathrm{HbA1c}$ level have been described in our previous report [21]. After adjusting for the intervention status, the improvements in $\mathrm{HbA} 1 \mathrm{c}$ during the two-year period of follow-up were significantly different by monthly income per capita and years of diabetes. No significant difference was observed in improvements by age, sex, educational level, tobacco and alcohol use, medications, HL level, and PA level (Table 2). 
Table 1. Physical activity (PA) levels during the study period among diabetes patients in four arms.

\begin{tabular}{|c|c|c|c|c|c|}
\hline \multirow{2}{*}{$\begin{array}{c}\text { Median (IQR) of PA } \\
(\mathrm{METs}-\mathrm{h} / \mathrm{w})\end{array}$} & \multicolumn{3}{|c|}{ Intervention Groups } & \multirow{2}{*}{ Control Arm } & \multirow{2}{*}{$p$-Value ${ }^{\mathrm{a}}$} \\
\hline & Health Literacy & Exercise & Comprehensive & & \\
\hline \multicolumn{6}{|l|}{ At baseline } \\
\hline Overall & $56.0(30.0,84.0)$ & $50.5(33.5,80.7)$ & $50.0(28.0,90.0)$ & $71.5(53.0,108.0)$ & $<0.001$ \\
\hline Exercise & $0(0,14.0)$ & $4.0(0,20.0)$ & $0(0,20.0)$ & $7.5(0,28.0)$ & 0.01 \\
\hline Commute & $18.7(0,30.2)$ & $16.0(8.0,28.0)$ & $12.0(0,28.0)$ & $28.0(14.0,29.0)$ & $<0.001$ \\
\hline Housework & $21.0(4.5,42.0)$ & $21.0(10.5,42.0)$ & $21.0(10.5,42.0)$ & $42.0(21.0,52.5)$ & $<0.001$ \\
\hline Sedentariness & $35.0(28.0,49.0)$ & $31.5(21.0,49.0)$ & $28.0(14.1,35.0)$ & $24.5(14.0,31.5)$ & $<0.001$ \\
\hline \multicolumn{6}{|l|}{ At 12 months } \\
\hline Overall & $41.8(16.9,66.1) *$ & $70.0(41.5,97.4) *$ & $55.0(28.0,81.0)$ & $73.7(44.3,108.3)$ & $<0.001$ \\
\hline Exercise & $0(0,10.0)$ & $12.0(0,22.7)$ & $8.2(0,28.0)$ & $7.3(0,28.0)$ & $<0.001$ \\
\hline Commute & $10.0(0,28.0)$ * & $23.3(10.0,38.5) *$ & $12.0(0,28.0)$ & $28.0(6.3,42.0)$ & $<0.001$ \\
\hline Housework & $18.0(5.0,42.0) *$ & $30.8(9.0,42.0)$ & $21.0(7.5,42.0)^{*}$ & $31.5(10.5,42.0)$ * & 0.001 \\
\hline Sedentariness & $28.0(21.0,35.0) *$ & $28.0(21.6,35.0) *$ & $28.0(21.0,35.0)$ & $28.0(21.0,42.0)$ * & 0.94 \\
\hline \multicolumn{6}{|l|}{ At 24 months } \\
\hline Overall & $39.5(17.8,69.4) *$ & $57.0(29.0,91.0)^{+}$ & $64.8(30.8,104.5)^{+}$ & $70.0(36.0,110.0)$ & $<0.001$ \\
\hline Exercise & $0(0,4.7)^{*+}$ & $4.0(0,20.0)^{+}$ & $17.0(0,38.0)^{*,+}$ & $8.0(0,24.0)$ & $<0.001$ \\
\hline Commute & $10.0(0,28.0)$ * & $18.7(8.0,42.0)$ * & $13.7(0,30.0)^{+}$ & $14.0(0,30.7)$ & $<0.001$ \\
\hline Housework & $21.0(6.0,42.0)^{+}$ & $21.0(9.0,42.0)^{+}$ & $21.0(9.0,42.0)$ * & $31.5(10.5,49.0)$ * & 0.03 \\
\hline Sedentariness & $35.0(21.0,42.0)^{*+}$ & $31.5(21.0,45.5)^{+}$ & $28.0(21.0,42.0) *$ & $28.0(17.5,35.0)$ * & $<0.001$ \\
\hline
\end{tabular}

PA: Physical Activity; IQR: interquartile range; METs-h/w: metabolic equivalents (METs)-hours per week. ${ }^{a}$ ANOVA tests (normal distributed) or Kruskal-Wallis tests (non-normal distributed) for group-comparisons; ${ }^{*} p<0.05$ for differences in PA level between baseline and 12 months and between baseline and 24 months within each group using a mixed linear model; ${ }^{+} p<0.05$ for difference in PA level between 12 and 24 months within each group using a mixed linear model.

Table 2. $\beta$ and $95 \% \mathrm{CI}$ of hemoglobin A1c (HbA1c) during the following-up with baseline characteristics of the study participants.

\begin{tabular}{|c|c|c|c|c|c|}
\hline \multirow{2}{*}{ Baseline Characteristics } & \multicolumn{3}{|c|}{ HbA1c Level (\%, Median, IQR) } & \multicolumn{2}{|c|}{$\beta$ Coefficients (SE) } \\
\hline & At baseline & At 12-months & At 24-months & Unadjusted & Adjusted $^{a}$ \\
\hline \multicolumn{6}{|l|}{ Age (years) } \\
\hline$<65$ & $8.1(7.5,9.0)$ & $8.0(6.9,9.1)$ & $7.9(7.0,9.1)$ & 0 (ref) & 0 (ref) \\
\hline$\geq 65$ & $8.1(7.6,9.2)$ & $8.0(7.1,9.1)$ & $7.9(6.9,9.1)$ & $0.05(0.10)$ & $0.06(0.14)$ \\
\hline \multicolumn{6}{|l|}{$\operatorname{Sex}(\%)$} \\
\hline Men & $8.1(7.6,9.1)$ & $8.1(7.1,9.2)$ & $7.9(7.0,9.1)$ & 0 (ref) & 0 (ref) \\
\hline Women & $8.1(7.5,9.1)$ & $8.0(7.1,9.1)$ & $7.8(6.9,9.1)$ & $-0.11(0.10)$ & $-0.11(0.09)$ \\
\hline \multicolumn{6}{|l|}{ Educational level (\%) } \\
\hline Primary school or below & $8.1(7.5,8.9)$ & $8.1(7.2,9.3)$ & $8.1(7.2,9.7)$ & 0 (ref) & 0 (ref) \\
\hline Junior high school & $8.2(7.6,9.3)$ & $8.1(7.1,9.2)$ & $7.9(7.0,9.1)$ & $-0.13(0.13)$ & $-0.23(0.13)$ \\
\hline Senior high school & $8.1(7.6,8.9)$ & $8.0(7.1,8.8)$ & $7.9(7.0,8.9)$ & $-0.20(0.14)$ & $-0.25(0.13)$ \\
\hline College and above & $7.9(7.4,8.7)$ & $7.6(6.7,9.1)$ & $7.5(6.7,8.6)$ & $-0.31(0.17)$ & $-0.29(0.16)$ \\
\hline \multicolumn{6}{|c|}{ Monthly income per capita (USD, \%) } \\
\hline$<308$ & $8.1(7.5,8.8)$ & $8.3(7.5,9.5)$ & $8.3(7.4,9.7)$ & 0 (ref) & 0 (ref) \\
\hline $308-769$ & $8.1(7.6,9.2)$ & $7.9(6.9,9.0)$ & $7.8(7.0,9.0)$ & $-0.32(0.14) *$ & $-0.40(0.13) * *$ \\
\hline$\geq 769$ & $8.0(7.5,9.2)$ & $8.0(7.2,9.1)$ & $7.7(6.9,8.8)$ & $-0.31(0.16) *$ & $-0.34(0.15) *$ \\
\hline \multicolumn{6}{|l|}{ Tobacco use $(\%)$} \\
\hline Never & $8.1(7.6,9.1)$ & $8.0(7.1,9.1)$ & $7.9(7.0,9.0)$ & 0 (ref) & 0 (ref) \\
\hline Ever & $8.0(7.5,9.2)$ & $8.2(7.1,9.3)$ & $8.2(7.1,9.4)$ & $0.09(0.14)$ & $-0.01(0.14)$ \\
\hline \multicolumn{6}{|l|}{ Alcohol drinking (\%) } \\
\hline Never & $8.1(7.6,9.1)$ & $8.0(7.0,9.1)$ & $7.9(6.9,9.1)$ & 0 (ref) & 0 (ref) \\
\hline Ever & $8.3(7.5,9.2)$ & $8.3(7.2,9.3)$ & $8.3(7.4,9.2)$ & $0.08(0.15)$ & $-0.01(0.15)$ \\
\hline \multicolumn{6}{|l|}{ Years of diabetes } \\
\hline$<10$ & $8.0(7.5,8.9)$ & $7.7(6.9,9.0)$ & $7.7(6.8,8.9)$ & 0 (ref) & 0 (ref) \\
\hline$\geq 10$ & $8.2(7.7,9.4)$ & $8.1(7.4,9.3)$ & $8.0(7.2,9.2)$ & $0.37(0.09) * *$ & $0.31(0.09)^{* *}$ \\
\hline Medications (\%) & & & & & \\
\hline
\end{tabular}


Table 2. Cont.

\begin{tabular}{|c|c|c|c|c|c|}
\hline \multirow{2}{*}{ Baseline Characteristics } & \multicolumn{3}{|c|}{ HbA1c Level (\%, Median, IQR) } & \multicolumn{2}{|c|}{$\beta$ Coefficients (SE) } \\
\hline & At baseline & At 12-months & At 24-months & Unadjusted & Adjusted $^{a}$ \\
\hline Diabetes pills only & $8.1(7.5,9.1)$ & $7.9(7.0,9.1)$ & $7.9(6.9,9.1)$ & $0.09(0.18)$ & $0.02(0.17)$ \\
\hline Insulin shot only & $8.1(7.6,9.5)$ & $8.4(7.3,9.0)$ & $8.0(7.3,9.2)$ & $0.10(0.12)$ & $0.06(0.11)$ \\
\hline Neither & $8.0(7.5,8.9)$ & $7.7(6.8,9.5)$ & $7.3(6.7,8.2)$ & 0 (ref) & 0 (ref) \\
\hline Both & $8.3(7.8,9.2)$ & $8.3(7.4,9.4)$ & $8.0(7.1,9.4)$ & $-0.19(0.21)$ & $-0.10(0.20)$ \\
\hline \multicolumn{6}{|l|}{ c-HeLMS score } \\
\hline$<116$ & $8.1(7.6,9.2)$ & $8.0(7.1,9.1)$ & $7.9(6.8,9.1)$ & 0 (ref) & 0 (ref) \\
\hline$\geq 116$ & $8.1(7.6,9.1)$ & $7.9(7.0,9.1)$ & $7.8(7.1,9.0)$ & $0.04(0.10)$ & $0.04(0.09)$ \\
\hline \multicolumn{6}{|l|}{ Correct rate of c-DNT-5 } \\
\hline$<80$ & $8.3(7.7,9.4)$ & $8.0(7.1,9.1)$ & $7.7(6.8,9.2)$ & 0 (ref) & 0 (ref) \\
\hline$\geq 80$ & $8.1(7.5,9.0)$ & $8.0(7.1,9.1)$ & $7.9(7.0,9.0)$ & $-0.01(0.11)$ & $0.11(0.10)$ \\
\hline \multicolumn{6}{|l|}{ PA level (Mets, by tertile) } \\
\hline$<42$ & $8.2(7.7,9.0)$ & $8.0(7.1,9.0)$ & $8.0(6.9,9.1)$ & 0 (ref) & 0 (ref) \\
\hline $42-78$ & $8.1(7.5,9.2)$ & $7.9(7.0,9.2)$ & $7.8(7.0,9.0)$ & $0.04(0.12)$ & $0.07(0.11)$ \\
\hline$\geq 78$ & $8.1(7.5,9.2)$ & $8.1(7.1,9.3)$ & $7.9(7.0,9.0)$ & $-0.05(0.12)$ & $-0.02(0.12)$ \\
\hline
\end{tabular}

SE: Standard Error; USD: USA dollar; HeLMS: Health Literacy Management Scale; c-DNT-5: the 5-item Diabetes Numeracy Test scale; $\beta$ derived from three-level mixed regression models (intervention status are level-3 observation units, individuals are level-2 and repeated measurements are level-1). ${ }^{a}$ adjusted for age, sex, and baseline $\mathrm{HbA1c}{ }^{*} p$ value $<0.05 ;{ }^{* *} p$ value $<0.01$.

As shown in Table 1, the overall PA level in control arm remained unchanged during the whole 2-year observation. In the exercise group, PA level was observed to increase from baseline to post-intervention $(p<0.001)$ but decreased significantly to the baseline level during the next 12 months $(p>0.05)$. In the HL group, the overall PA level decreased significantly from baseline to post-intervention $(p<0.001)$ and remained unchanged thereafter. The PA level did not change significantly during the 1-year intervention in the comprehensive group, but it increased significantly during the 1-year post-intervention.

We reclassified all participants into three groups according to tertile of PA levels at 12 months (at the end of 1-year intervention) and at 24 months (1-year post-intervention), respectively. As presented in Table 3, the three groups classified by PA level at two time points differed in age, sex, alcohol drinking, hypoglycemic medications, and PA level at baseline.

Further analysis show a significant improvement in $\mathrm{HbA1c}$ for the medium versus the lowest tertile group of PA level at 12 months ( $\beta$ : $-3.47,95 \% \mathrm{CI}:-5.33,-1.60)$ and for the highest versus the lowest tertile group of PA at 24 months ( $\beta$ : $-0.50,95 \% \mathrm{CI}:-1.00,-0.01)$ after adjusting for potential confounders including significant interaction of PA with initial levels of $\mathrm{HbA} 1 \mathrm{c}$ and PA (Table 4). As a result, during the whole 2-year period, a significant improvement in $\mathrm{HbA} 1 \mathrm{c}$ was observed for the highest versus the lowest tertile group of PA ( $\beta$ : $-3.49 ; 95 \% \mathrm{CI}:-5.87,-1.11)$, and a significant interaction was found for PA with baseline levels of PA and $\mathrm{HbA1c}$ ( $p$ for interaction $<0.05$ ). 
Table 3. Baseline characteristics of the study participants by tertile groups of PA at the follow-up surveys.

\begin{tabular}{|c|c|c|c|c|c|c|c|c|}
\hline \multirow[b]{2}{*}{ Characteristics at Baseline } & \multicolumn{3}{|c|}{ PA Level at the 12-Month Survey (METs-h/w) } & \multirow[b]{2}{*}{$p$-Values } & \multicolumn{3}{|c|}{ PA Level at the 24-Month Survey (METs-h/w) } & \multirow[b]{2}{*}{$p$-Values } \\
\hline & $\begin{array}{l}\text { Tertile Group } 1 \\
(<38.7, n=236)\end{array}$ & $\begin{array}{c}\text { Tertile Group } 2 \\
(38.7 \text { to } 77.0, n=233)\end{array}$ & $\begin{array}{l}\text { Tertile Group } 3 \\
(\geq 77.0, n=235)\end{array}$ & & $\begin{array}{l}\text { Tertile Group } 1 \\
(<38.8, n=218)\end{array}$ & $\begin{array}{c}\text { Tertile Group } 2 \\
(38.8 \text { to } 82.0, n=214)\end{array}$ & $\begin{array}{l}\text { Tertile Group } 3 \\
(\geq 82.0, n=220)\end{array}$ & \\
\hline Age (years, median, IQR) a & $67(60,74)$ & $67(60,72)$ & $64(58,69)$ & $<0.001$ & $68(60,74)$ & $66(59,70)$ & $64(59,70)$ & 0.002 \\
\hline Educational level (\%) ${ }^{\mathrm{b}}$ & & & & 0.118 & & & & 0.432 \\
\hline Primary school or below & 25.1 & 26.6 & 15.7 & & 27.2 & 18.7 & 20.9 & \\
\hline Junior high school & 35.3 & 39.1 & 40.9 & & 34.5 & 39.7 & 40.0 & \\
\hline Senior high school & 26.4 & 19.3 & 29.4 & & 24.9 & 25.2 & 25.5 & \\
\hline College and above & 12.2 & 15.0 & 14.0 & & 13.4 & 16.4 & 13.6 & \\
\hline$<308$ & 13.5 & 18.7 & 11.5 & & 20.0 & 11.7 & 12.9 & \\
\hline $308-769$ & 53.0 & 59.6 & 61.3 & & 49.8 & 58.7 & 62.7 & \\
\hline$>769$ & 33.5 & 21.7 & 27.2 & & 30.2 & 29.6 & 24.4 & \\
\hline Tobacco smoking (\%) ${ }^{\mathrm{b}}$ & 18.8 & 13.4 & 14.6 & 0.210 & 15.3 & 14.6 & 14.3 & 0.771 \\
\hline Alcohol drinking $(\%)^{\mathrm{b}}$ & 14.2 & 11.9 & 8.3 & 0.045 & 16.6 & 10.1 & 7.6 & 0.003 \\
\hline Years of diabetes (median, IQR) ${ }^{a}$ & $10.0(5.3,16.4)$ & $9.8(5.0,14.8)$ & $9.4(4.8,15.1)$ & 0.362 & $11.0(5.5,17.0)$ & $8.9(4.8,14.9)$ & $9.7(5.0,15.0)$ & 0.026 \\
\hline \multicolumn{2}{|c|}{ Use of anti-diabetes agents and insulin $(\%)^{\mathrm{b}}$} & & & 0.024 & & & & 0.660 \\
\hline Diabetes pills only & 61.9 & 67.2 & 52.1 & & 62.2 & 64.4 & 61.5 & \\
\hline Insulin shot only & 10.2 & 4.9 & 9.3 & & 10.1 & 7.3 & 8.9 & \\
\hline Both & 24.8 & 23.0 & 49.4 & & 22.0 & 23.4 & 22.1 & \\
\hline $\mathrm{HbA} 1 \mathrm{c}$ level $(\%)^{\mathrm{b}}$ & $8.2(7.6,9.2)$ & $8.1(7.5,9.0)$ & $8.2(7.5,9.3)$ & 0.655 & $8.2(7.6,9.2)$ & $8.2(7.5,9.2)$ & $8.1(7.6,8.9)$ & 0.567 \\
\hline $\mathrm{HbA} 1 \mathrm{c}<7.0 \%(\%)^{\mathrm{b}}$ & 22.5 & 22.3 & 21.7 & 0.844 & 26.2 & 24.3 & 25.9 & 0.956 \\
\hline Energy Intake (Kcal, median, IQR) ${ }^{a}$ & $1448(1116,1793)$ & $1522(1197,1826)$ & $1380(1130,1691)$ & 0.120 & $1467(1134,1821)$ & $1433(1132,1720)$ & $1410(1141,1711)$ & 0.384 \\
\hline
\end{tabular}

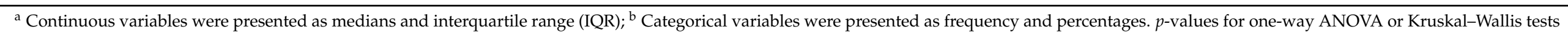

(continuous variables) and chi-square tests (categorical variables). 
Table 4. Associations of PA level with improvements in HbA1c among the study participants.

\begin{tabular}{|c|c|c|c|c|c|c|c|}
\hline \multirow{2}{*}{ Associations of PA level with $\mathrm{HbA1c}$} & \multicolumn{3}{|c|}{ Tertile Groups by PA Level (METs-h/w) ${ }^{a}$} & \multirow{2}{*}{$p$-Values } & \multicolumn{2}{|c|}{ Achievement of Recommended Exercise Level } & \multirow{2}{*}{$p$-Values } \\
\hline & Lowest & Medium & Highest & & No & Yes & \\
\hline \multicolumn{8}{|l|}{ At 12 months } \\
\hline Number of subjects & 236 & 233 & 235 & & 391 & 313 & \\
\hline HbA1c < 7.0\% $(n, \%)$ & $53(22.5)$ & $52(22.3)$ & $51(21.7)$ & 0.844 & $87(22.3)$ & $69(22.0)$ & 0.948 \\
\hline$\beta 1(95 \% \mathrm{CI})$ & 0 (ref) & $0.12(-0.16,0.40)$ & $0.15(-0.15,0.45)$ & & 0 (ref) & $-0.15(-0.39,0.09)$ & \\
\hline$\beta 2(95 \% \mathrm{CI})$ & 0 (ref) & $0.15(-0.12,0.42)$ & $0.06(-0.23,0.35)$ & & 0 (ref) & $-0.05(-0.29,0.18)$ & \\
\hline$\beta 3(95 \% \mathrm{CI})^{\text {a }}$ & 0 (ref) & $-3.47(-5.33,-1.60)$ & $-0.85(-2.58,0.88)$ & & 0 (ref) & $-0.28(-0.54,-0.02)$ & \\
\hline \multicolumn{8}{|l|}{ At 24 months } \\
\hline HbA1c (\%, median, IQR) & $8.0(6.9,9.1)$ & $7.9(7.0,8.9)$ & $7.8(6.9,9.0)$ & 0.794 & $7.8(6.3,9.0)$ & $7.9(6.9,9.1)$ & 0.816 \\
\hline $\mathrm{HbA} 1 \mathrm{c}<7.0 \%(n, \%)$ & $57(26.2)$ & $52(24.3)$ & $57(25.9)$ & 0.956 & $94(25.3)$ & $72(25.7)$ & 0.897 \\
\hline$\beta 1(95 \% \mathrm{CI})$ & 0 (ref) & $-0.01(-0.31,0.29)$ & $-0.17(-0.47,0.14)$ & & 0 (ref) & $-0.07(-0.33,0.18)$ & \\
\hline$\beta 2(95 \% \mathrm{CI})$ & 0 (ref) & $0.03(-0.25,0.31)$ & $-0.10(-0.39,0.19)$ & & 0 (ref) & $-0.01(-0.25,0.23)$ & \\
\hline \multirow{2}{*}{\multicolumn{8}{|c|}{ From baseline to 24 months }} \\
\hline & & & & & & & \\
\hline$\beta(95 \% \mathrm{CI})$ & 0 (ref) & $-1.29(-3.33,0.75)$ & $-3.49(-5.87,-1.11)$ & & 0 (ref) & $-0.20(-0.38,-0.02)$ & \\
\hline
\end{tabular}

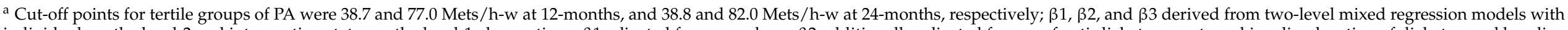

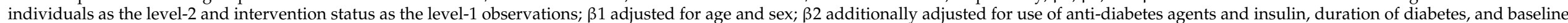

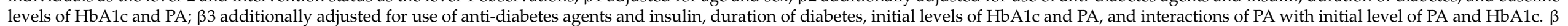

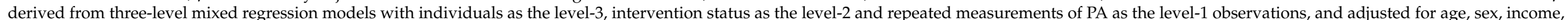

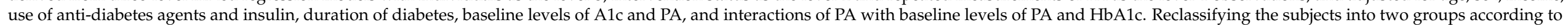

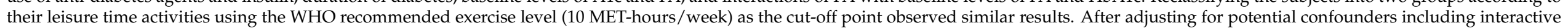

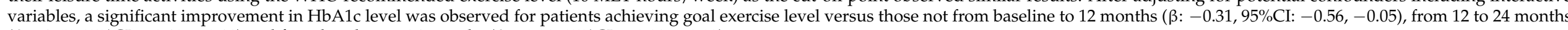
$(\beta:-1.65,95 \% \mathrm{CI}:-3.12,-0.18)$ and from baseline to 24 months ( $\beta$ : $-0.20,95 \% \mathrm{CI}:-0.38,-0.02)$. 
Figure 2 shows the potential effect of specific type of PA on HbA1c level. After adjusting for overall PA and other potential confounders, a significant increase in $\mathrm{HbA} 1 \mathrm{c}$ was observed for the highest versus the lowest tertile group of housework from baseline to 12 months ( $\beta$ : 0.47, 95\%CI: 0.07, 0.86). No significant association was observed for other type of PA with $\mathrm{HbA1c}$ level.

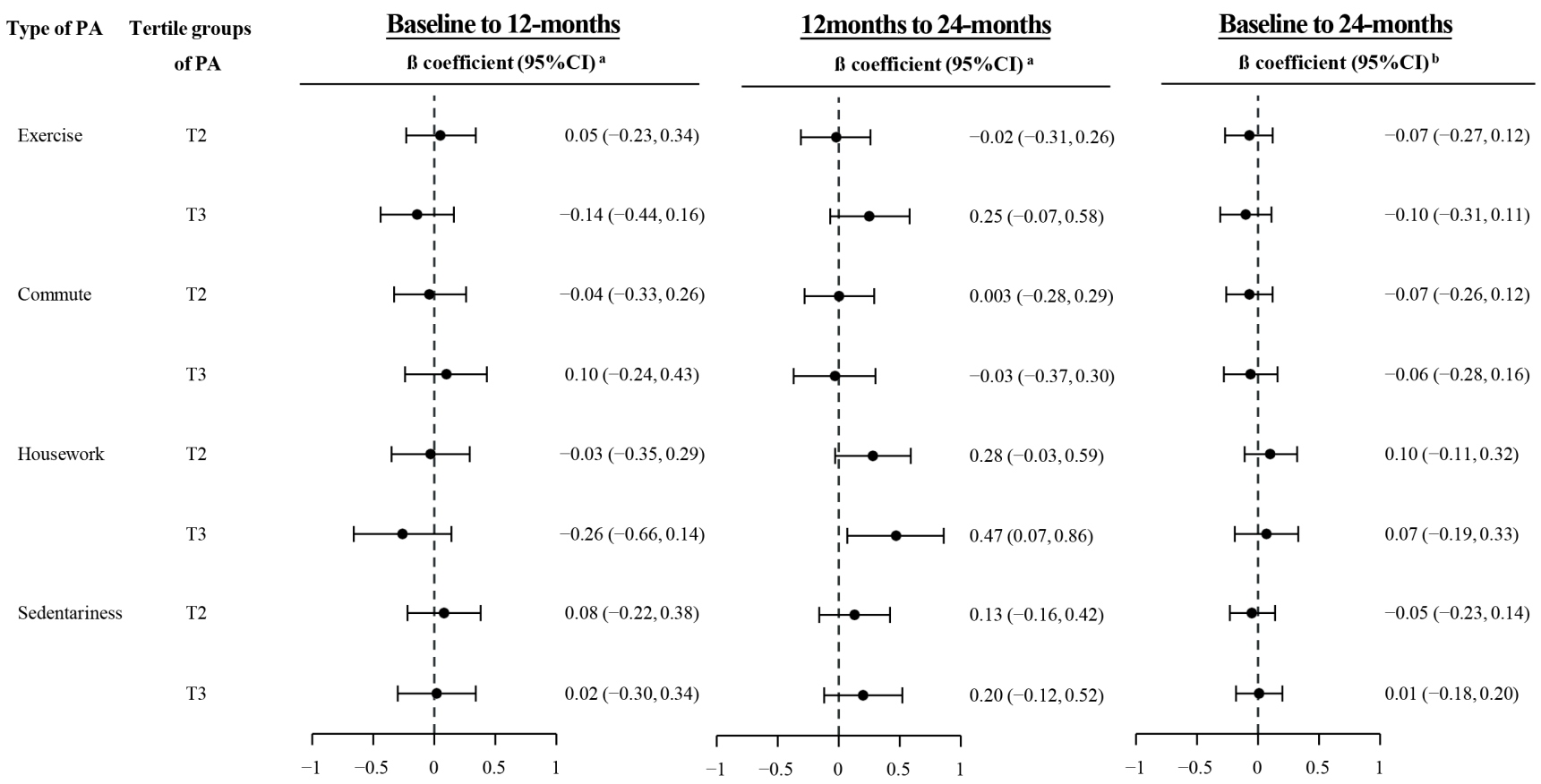

Figure 2. Associations of HbA1c with levels of specific type of PA (Physical Activity). ${ }^{\text {a }}$ Two-level mixed regression models with individuals as the level-2 and intervention status as the level-1 observations, and adjusted for age, sex, anti-diabetes drug use or insulin use, duration of diabetes, initial levels of A1c and PA, overall PA level; ${ }^{b}$ Three-level mixed regression models with individuals as the level-3, intervention status as the level-2, and repeated measurements of PA as the level-1 observations, and adjusted for age, sex, anti-diabetes drug or insulin use, duration of diabetes, baseline levels of A1c and PA, and overall PA level.

Stratified analyses by the medians of baseline $\mathrm{HbA} 1 \mathrm{c}$ and PA levels were conducted to demonstrate the potential joint effect of PA with the two factors. As shown in Figure 3, the associations of PA with HbA1c level differed by baseline levels of PA and/or HbA1c (all $p$ values for interaction $<0.05)$. An improved $\mathrm{HbA1c}$ level was associated with a higher level of PA only among patients with lower levels of baseline PA ( $<59.5$ MET-hours/week) and $\mathrm{HbA1c}(\geq 8.1 \%)$, with $\beta(95 \% \mathrm{CI})$ for the medium and the highest tertile groups versus the lowest group being $-0.21(-0.64,0.22)$ and $-0.05(-0.54,0.44)$ from baseline to 12 months, and being $-0.23(-0.57,0.12)$ and $-0.30(-0.68,0.09)$ from baseline to 24 months. In other groups with a higher baseline $\mathrm{PA}$ or $\mathrm{HbA1c}$ level, a positive association was observed between PA and HbA1c level. 


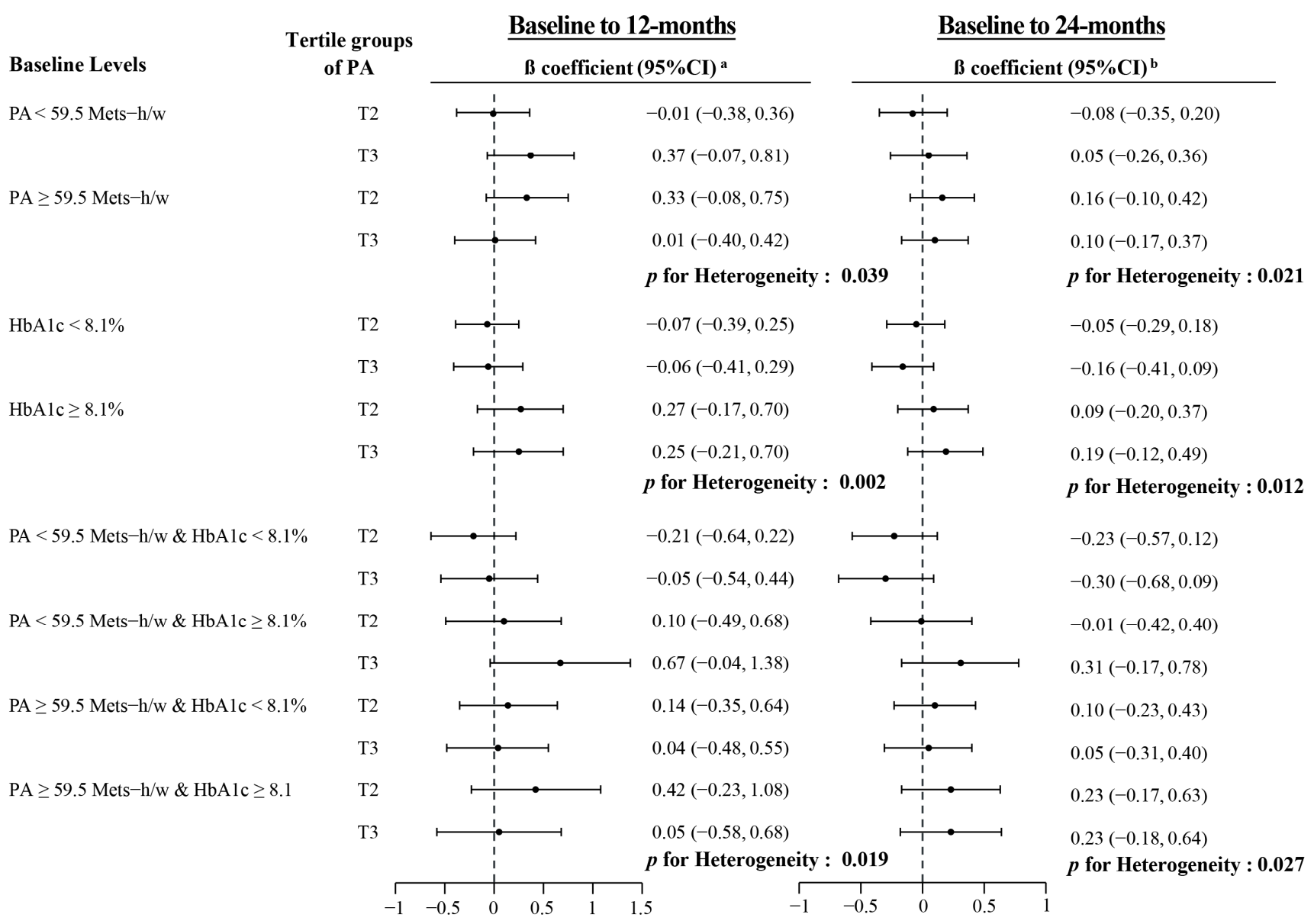

Figure 3. $\beta$ coefficients and 95\% confidence intervals for PA with HbA1c at 12 and 24 months by initial levels of PA and HbA1c. ${ }^{a}$ Two-level mixed regression models with individuals as the level-2 observations and intervention status as shown in Table 1 and adjusted for age, sex, use of anti-diabetes agents and insulin, duration of diabetes, and initial PA level (for stratified analysis by initial HbA1c only) or initial HbA1c level (for stratified analysis by initial PA only); ${ }^{b}$ Three-level mixed regression models with individuals as the level-3, intervention status as the level-2 and repeated measurements of PA as the level-1 observations, and adjusted for age, sex, use of anti-diabetes agents and insulin, duration of diabetes, and initial PA level (for stratified analysis by initial HbA1c only) or initial HbA1c level (for stratified analysis by initial PA only).

\section{Discussion}

In this secondary analysis of data from an RCT including community-dwelling Chinese diabetes patients, we compared the effectiveness of the exercise, HL, and comprehensive interventions on PA levels from three major domains, and we further evaluated the potential effect of PA on $\mathrm{HbA1c}$ level in the population. Our results suggest that an exercise-focused intervention may increase the PA level in Chinese diabetes patients, and the effect of PA on HbA1c depends on the baseline level of PA and/or HbA1c.

At the beginning of the interventions, 39.9\% of our patients met the 2018 exercise guidelines for people with diabetes, which recommends at least $150 \mathrm{~min}$ of moderate- to vigorous intensity aerobic exercise each week (10 MET-hours/week). As a result, the mean and median levels of baseline PA in our subjects were 67.9 and 59.5 MET-hours/week (4075 and 3570 MET-mins/week), respectively, which is much lower than 213 MET-hours/week (12,780 MET-mins/week), the average national level in Chinese adults in 2009. The sedentary lifestyles in our subjects may account, at least in part, for their poor glycemic control status.

Most previous studies observed the role of exercise intervention in increasing PA levels in T2DM patients [33-35], but a null result was usually observed for a health education intervention [36]. For instance, Araizi et al. [35] reported a significantly increased PA level 
in the active group who were instructed to walk at least 10,000 steps per day 5 or more days per week comparing with the control group in a 6-week RCT. Olson et al. [33] found that the aerobic exercise (walking) intervention increased PA level at post-intervention (month-two), but this was followed by a decline at six months. They did not observe any change in PA level across the period in an education group who were provided with an 8-week online diabetes and health education course. Consistent with these studies, we did not observe changes in PA level in the HL intervention group but found that an exercise-focused intervention-supervised walking-increased PA level in our subjects. However, the effect was not sustained during the post-intervention period, suggesting that continuous exercise interventions may be needed to maintain PA level in the population. Our results, as well as Olson et al.'s report [33], suggest the limited effect of health education on individuals' PA behaviors and the importance of supervised behavior interventions.

Interestingly, although HL intervention alone did not improve PA level, the comprehensive group receiving both HL and PA interventions had a higher PA level during the post-intervention period, suggesting the long-term effect of combined use of exercise and HL interventions. It seems that HL intervention may help to promote autonomous motives [37] and increase adherence to exercise intervention in our subjects. The adherence was influenced not only by the intervention package used but also by the factors such as social support, physical fitness, socioeconomic status, knowledge of diabetes, infrastructure provision, and integrated policies [38-40]. Therefore, continuous exercise intervention or combined use of exercise and HL interventions should be provided to diabetes patients to establish healthy behaviors for long-term benefits.

Diabetes patients are usually encouraged to be active, which has been beneficial for glycemic control. In a meta-analysis, Avery et al. [41] demonstrated a significant negative relationship between increase in PA intensity and $\mathrm{HbA1c}$ level, highlighting the importance of physically active behaviors. However, evidence was far from consistent. Mynarski et al. [42] did not find a significant correlation between $\mathrm{HbA1c}$ level and PA level in T2DM patients. Based on the observed limited impact of PA on glycemic/metabolic control, Wisse et al. [43] made a conclusion that a PA program was not sufficient to improve glycemic control in T2DM patients. It seems that the functional thresholds, in terms of both the volume and intensity of the delivered PA intervention, may exist and are needed for glucose control. Van Dyck et al. [44] found that HbA1c was improved significantly only among diabetes patients who increased more than 4000 steps per day. In a cross-sectional study, Li et al. [45] found that HbA1c was significantly lower in the sufficiently active group (10-50 Met-hours/week) and very active group ( $>50$ Met-hours/week) than in the insufficient active group ( $<10$ Met-hours/week).

In this study, we observed a significant negative association between PA and $\mathrm{HbA1c}$ only after taking initial levels $\mathrm{PA}$ and $\mathrm{HbA} 1 \mathrm{c}$ into consideration. In stratified analysis by baseline levels of PA and $\mathrm{HbA1c}$, we observed a negative association between PA and $\mathrm{HbA} 1 \mathrm{c}$ level only among patients with a lower level of baseline PA and $\mathrm{HbA1c}$. It appears that patients with more than 59.5 MET-hours/week of PA had already reached the functional threshold of PA and could not benefit more from an additional increase in PA in glycemic control.

The strengths of this study included the longitudinal design and large sample size, which enable us to evaluate the effect of PA directly, and made it possible to conduct stratified analysis by initial PA and HbA1c level. Second, the measurement of PA across a broad spectrum of daily living domains enabled us to evaluate the effect of total PA and specific type of PA, while most previous studies just focused on the effect of leisure-time activities. Moreover, this study is among the few studies evaluating the post-intervention effects of PA on $\mathrm{HbA} 1 \mathrm{c}$ in diabetes patients, providing evidence on the long-term beneficial effect of PA. Finally, the use of multiple level mixed regression models enables us to evaluate the independent effect of PA on $\mathrm{HbA1c}$ during the whole period of follow-up.

Several limitations should be mentioned. First, the baseline levels of PA and $\mathrm{HbA1c}$ were not comparable across the groups reclassified by PA, which may have introduced 
confounding effects to the results. However, stratified analyses by the two factors not only released our concern on their confounding effects but also demonstrated their potential modifying effects on PA-HbA1c associations. Second, PA level was measured based on the IPAQ, and the METs were calculated based on the average level of PA regardless of age, sex, body size, and environmental conditions in which the activities were performed. Moreover, PA level was measured repeatedly using IPAQ, which is not reliable to measure the changes in PA at the individual level [46]. Misclassification bias could not be avoided. Finally, detailed information on anti-diabetes agent use and insulin shots were not available across the whole period of follow-up. Residual confounding effect of the factors, as well as other factors such as diet and self-efficacy, could not be excluded.

\section{Conclusions}

In summary, PA level can be improved by exercise-focused intervention in Chinese T2DM patients. The benefit effect of PA on $\mathrm{HbA1c}$ level is dependent on baseline levels of $\mathrm{PA}$ and $\mathrm{HbA1c}$. Our results, particularly the beneficial role of PA in improving $\mathrm{HbA} 1 \mathrm{c}$ in patients with lower levels of PA or HbA1c, suggest that the interventions should be addressed in the physically inactive patients to improve their PA level to a functional threshold.

Supplementary Materials: The following are available online at https://www.mdpi.com/article/10 .3390 /ijerph18084292/s1, Table S1: Baseline characteristics of the study participants by intervention status; Table S2: Baseline intensity of PA by HbA1c levels in the study participants, Figure S1: Exercise module in the PRIDE toolkit.

Author Contributions: W.-Y.Y. and M.-G.H. drafted the manuscript. H.F., Q.X. and X.L. contributed to data collection and quality control. G.D.V., Y.C. and Y.W. contributed to statistical analysis. W.-H.X. and G.D.V. designed the exercise intervention package. W.-H.X. and R.L.R. contributed to study design and statistical analysis. All authors contributed to the interpretation of data and revision of the manuscript. All authors have read and agreed to the published version of the manuscript.

Funding: This work was supported by the National Nature Science Foundation of China (81773504), the China Medical Board (CMB) Open Competition Project (No.13-159) and the Social Science Fund of China National Ministry of Education (No. 14YJAZH092).

Institutional Review Board Statement: Ethics approval was obtained from the Medical Ethics Committee of Fudan University (IRB00002408 \& FWA00002399) before recruiting study participants (registration number: 2013-06-0451). All local medical ethics committees agreed with this approval.

Informed Consent Statement: All participants provided written informed consent prior to the start of the study.

Data Availability Statement: The datasets used and/or analyzed during the current study are available from the corresponding author on reasonable request.

Acknowledgments: The authors thank the participants of this study and the support of healthcare providers in involved Community Healthcare Centers in Minhang and Changning districts of Shanghai, China.

Conflicts of Interest: The authors declare that they have no competing interests.

\section{References}

1. World Health Organization. Global Report on Diabetes; World Health Organization: Geneva, Switzerland, 2016.

2. Shigaki, C.; Kruse, R.L.; Mehr, D.; Sheldon, K.M.; Ge, B.; Moore, C.; Lemaster, J. Motivation and diabetes self-management. Chronic Illn. 2010, 6, 202-214. [CrossRef] [PubMed]

3. O'Hagan, C.; De Vito, G.; Boreham, C.A. Exercise prescription in the treatment of type 2 diabetes mellitus: Current practices, existing guidelines and future directions. Sports Med. 2013, 43, 39-49. [CrossRef] [PubMed]

4. Caspersen, C.J.; Powell, K.E.; Christenson, G.M. Physical activity, exercise, and physical fitness: Definitions and distinctions for health-related research. Public Health Rep. 1985, 100, 126-131.

5. Dutton, G.R.; Lewis, C.E. The Look AHEAD Trial: Implications for lifestyle intervention in type 2 diabetes mellitus. Prog. Cardiovasc. Dis. 2015, 58, 69-75. [CrossRef] 
6. Blomster, J.I.; Chow, C.K.; Zoungas, S.; Woodward, M.; Patel, A.; Marre, M.; Harrap, S.; Chalmers, J.; Hilis, G.S. The influence of physical activity on vascular complications and mortality in patients with type 2 diabetes mellitus. Diabetes Obes. Metab. 2013, 15, 1008-1012. [CrossRef]

7. 2018 Physical Activity Guidelines Advisory Comittee. The 2018 Physical Activity Guidelines Advisory Committee Scientific Report; U.S. Department of Health and Human Services: Washington, DC, USA, 2018; p. 779.

8. Liu, H.; Liu, Z.; Wang, Y.; Stinchcombe, T.E.; Owzar, K.; Han, Y.; Hung, R.J.; Brhane, Y.; McLaughin, J.; Brennan, P.; et al. Functional variants in DCAF4 Associated with lung cancer risk in European populations. Carcinogenesis 2017, 38, 541-551. [CrossRef]

9. Monda, K.L.; Adair, L.S.; Zhai, F.; Popkin, B.M. Longitudinal relationships between occupational and domestic physical activity patterns and body weight in China. Eur. J. Clin. Nutr. 2008, 62, 1318-1325. [CrossRef]

10. Chao, D.; Foy, C.G.; Farmer, D. Exercise adherence among older adults: Challenges and strategies. Control. Clin. Trials 2000, 21, 212s-217s. [CrossRef]

11. Byrne, H.; Caulfield, B.; De Vito, G. Effects of self-directed exercise programmes on individuals with type 2 diabetes mellitus: A systematic review evaluating their effect on $\mathrm{HbA1c}$ and other metabolic outcomes, physical characteristics, cardiorespiratory fitness and functional outcomes. Sports Med. 2017, 47, 717-733. [CrossRef]

12. Physical Activity Guidelines Advisory Committee. Physical Activity Guidelines Advisory Committee Report; U.S. Department of Health and Human Services: Washington, DC, USA, 2008. Available online: https:/ /www.europarc.org/wp-content/uploads/ 2018/03/Physical-Activity-Guidelines-Advisory-Committee-Report-2008.pdf (accessed on 10 September 2012).

13. Yu, R.B.; Hong, X.; Ding, W.-L.; Tan, Y.-F.; Zhang, Y.-X.; Sun, N.-X.; Wu, G.-L.; Zhan, S.-W.; Ge, D.-F. The association between the genetic polymorphism of HLA-DQA1, DQB1, and DRB1 and serum alanine aminotransferase levels in chronic hepatitis C in the Chinese population. J. Gastroenterol. Hepatol. 2008, 23, 1394-1402. [CrossRef]

14. Nwasuruba, C.; Khan, M.; Egede, L.E. Racial/ethnic differences in multiple self-care behaviors in adults with diabetes. J. Gen. Intern. Med. 2007, 22, 115-120. [CrossRef]

15. Thiel, D.M.; Sayah, F.A.; Vallance, J.K.; Johnson, S.T.; Johnson, J.A. Association between physical activity and health-related quality of life in adults with type 2 diabetes. Can. J. Diabetes 2017, 41, 58-63. [CrossRef]

16. Xu, F.; Wang, Y.; Ware, R.S.; Tse, L.A.; Dunstan, D.W.; Liang, Y.; Wang, Z.; Hong, X.; Owen, N. Physical activity, family history of diabetes and risk of developing hyperglycaemia and diabetes among adults in mainland China. Diabet. Med. 2012, 29, 593-599. [CrossRef]

17. Rossen, J.; Yngve, A.; Hagströmer, M.; Brismar, K.; Ainsworth, B.E.; Iskull, C.; Möller, P.; Johansson, U.-B. Physical activity promotion in the primary care setting in pre- and type 2 diabetes-The Sophia Step Study, an RCT. BMC Public Health 2015, 15, 647. [CrossRef]

18. Nielsen-Bohlman, L.; Panzer, A.M.; Kindig, D.A. Health Literacy: A Prescription to End Confusion; National Academies Press (US): Washington, DC, USA, 2004.

19. Lam, M.H.; Leung, A.Y. The effectiveness of health literacy oriented programs on physical activity behaviour in middle aged and older adults with type 2 diabetes: A systematic review. Health Psychol. Res. 2016, 4, 5595. [CrossRef]

20. Rothman, R.L.; DeWalt, D.A.; Malone, R.; Bryant, B.; Shintani, A.; Crigler, B.; Weinberger, M.; Pignone, M. Influence of patient literacy on the effectiveness of a primary care-based diabetes disease management program. JAMA 2004, 292, 1711-1716. [CrossRef]

21. Wang, L.; Fang, H.; Xia, Q.; Liu, X.; Chen, Y.; Zhou, P.; Yan, Y.; Yao, B.; Wei, Y.; Jiang, Y.; et al. Health literacy and exercise-focused interventions on clinical measurements in Chinese diabetes patients: A cluster randomized controlled trial. EClinicalMedicine 2019, 17, 100211. [CrossRef]

22. Xu, W.H.; Rothman, R.L.; Li, R.; Chen, Y.; Xia, Q.; Fang, H.; Gao, J.; Yan, Y.; Zhou, P.; Jiang, Y. Improved self-management skills in Chinese diabetes patients through a comprehensive health literacy strategy: study protocol of a cluster randomized controlled trial. Trials 2014, 15, 498. [CrossRef]

23. Turner, L.; Shamseer, L.; Altman, D.G.; Weeks, L.; Peters, J.; Kober, T.; Dias, S.; Schulz, K.F.; Plint, A.C.; Moher, D. Consolidated Standards of Reporting Trials (CONSORT) and the Completeness of Reporting of Randomised Controlled trials (RCTs) published in medical journals. Cochrane Database Syst. Rev. 2012, 11, MR000030. [CrossRef]

24. Chinese Diabetes Society. Standards of care for type 2 diabetes in China (2013). Chin J Diabetes. 2014, 8, 2-42. (in Chinese).

25. Borg, G. Psychophysical scaling with applications in physical work and the perception of exertion. Scand J. Work Environ. Health 1990, 16 (Suppl. 1), 55-58. [CrossRef] [PubMed]

26. Wolff, K.; Chambers, L.; Bumol, S.; White, R.O.; Gregory, B.P.; Davis, D.; Rothman, R.L. The PRIDE (Partnership to Improve Diabetes Education) Toolkit: Development and evaluation of novel literacy and culturally sensitive diabetes education materials. Diabetes Educ. 2016, 42, 23-33. [CrossRef]

27. Wolff, K.; Cavanaugh, K.; Malone, R.; Hawk, V.; Gregory, B.P.; Davis, D.; Wallston, K.; Rothman, R. The Diabetes Literacy and Numeracy Education Toolkit (DLNET): Materials to facilitate diabetes education and management in patients with low literacy and numeracy skills. Diabetes Educ. 2009, 35, 233-236, 238-241, 244-245. [CrossRef]

28. Department of Noncommunicable Disease and Mental Health World Health Organization. Stepwise Surveillance for Chronic Non-Communicable Diseases (STEPS) I; World Health Organization: Geneva, Switzerland, 2010. Available online: https://www. who.int/ncds/surveillance/steps/GPAQ_CH.pdf (accessed on 10 September 2012). (In Chinese) 
29. Limb, E.S.; Ahmad, S.; Cook, D.G.; Kerry, S.M.; Ekelund, U.; Whincup, P.H.; Victor, C.R.; Iliffe, S.; Ussher, M.; Fox-Rushby, J.; et al. Measuring change in trials of physical activity interventions: A comparison of self-report questionnaire and accelerometry within the PACE-UP Trial. Int. J. Behav. Nutr. Phys. Act. 2019, 16, 10. [CrossRef] [PubMed]

30. Ainsworth, B.E.; Haskell, W.L.; Leon, A.S.; Jacobs, D.R., Jr.; Montoye, H.J.; Sallis, J.F.; Paffenbarger, R.S. Compendium of physical activities: Classification of energy costs of human physical activities. Med. Sci. Sports Exerc. 1993, 25, 71-80. [CrossRef] [PubMed]

31. Chu, A.H.; Ng, S.H.X.; Koh, D.; Müller-Riemenschneider, F. Reliability and validity of the self- and interviewer-administered versions of the Global Physical Activity Questionnaire (GPAQ). PLoS ONE 2015, 10, e0136944.

32. World Health Organization. Global Physical Activity Questionnaire (GPAQ) Analysis Guide; World Health Organization: Geneva, Switzerland, 2003.

33. Olson, E.A.; McAuley, E. Impact of a brief intervention on self-regulation, self-efficacy and physical activity in older adults with type 2 diabetes. J. Behav. Med. 2015, 38, 886-898. [CrossRef]

34. Melkus, G.D. A 12-month intensive supervised exercise intervention and counselling reduces HbA1c, blood pressure and other modifiable cardiovascular risk factors in people with type 2 diabetes. Evid. Based Nurs. 2011, 14, 68-69. [CrossRef]

35. Araiza, P.; Hewes, H.; Gashetewa, C.; Vella, C.A.; Burge, M.R. Efficacy of a pedometer-based physical activity program on parameters of diabetes control in type 2 diabetes mellitus. Metabolism 2006, 55, 1382-1387. [CrossRef]

36. Rosal, M.C.; Ockene, I.S.; Restrepo, A.; White, M.J.; Borg, A.; Olendzki, B.; Scavron, J.; Candib, L.; Welch, G.; Reed, G. Randomized trial of a literacy-sensitive, culturally tailored diabetes self-management intervention for low-income Latinos: Latinos en control. Diabetes Care 2011, 34, 838-844. [CrossRef]

37. Miquelon, P.; Castonguay, A. Motives for participation in physical activity and observance of physical activity recommendations among adults with type 2 diabetes. Can. J. Diabetes 2016, 40, 399-405. [CrossRef] [PubMed]

38. Parajuli, J.; Saleh, F.; Thapa, N.; Ali, L. Factors associated with nonadherence to diet and physical activity among nepalese type 2 diabetes patients; a cross sectional study. BMC Res. Notes 2014, 7, 758. [CrossRef] [PubMed]

39. Ng, S.W.; Popkin, B.M. Time use and physical activity: A shift away from movement across the globe. Obes. Rev. 2012, 13, 659-680. [CrossRef] [PubMed]

40. Murano, I.; Asakawa, Y.; Mizukami, M.; Takihara, J.; Shimizu, K.; Imai, T. Factors increasing physical activity levels in diabetes mellitus: A survey of patients after an inpatient diabetes education program. J. Phys. Ther. Sci. 2014, 26, 695-699. [CrossRef]

41. Avery, L.; Flynn, D.; Dombrowski, S.U.; van Wersch, A.; Sniehotta, F.F.; Trenell, M.I. Successful behavioural strategies to increase physical activity and improve glucose control in adults with type 2 diabetes. Diabet. Med. 2015, 32, 1058-1062. [CrossRef]

42. Mynarski, W.; Psurek, A.; Borek, Z.; Rozpara, M.; Grabara, M.; Strojek, K. Declared and real physical activity in patients with type 2 diabetes mellitus as assessed by the international physical activity questionnaire and caltrac accelerometer monitor: A potential tool for physical activity assessment in patients with type 2 diabetes mellitus. Diabetes Res. Clin. Pract. 2012, 98, 46-50.

43. Wisse, W.; Rookhuizen, M.B.; de Kruif, M.D.; van Rossum, J.; Jordans, I.; ten Cate, H.; van Loon, L.J.C.; Meesters, E.W. Prescription of physical activity is not sufficient to change sedentary behavior and improve glycemic control in type 2 diabetes patients. Diabetes Res. Clin. Pract. 2010, 88, e10-3. [CrossRef]

44. Van Dyck, D.; De Greef, K.; Deforche, B.; Ruige, J.; Bouckaert, J.; Tudor-Locke, C.E.; Kaufman, J.-M.; De Bourdeaudhuij, I. The relationship between changes in steps/day and health outcomes after a pedometer-based physical activity intervention with telephone support in type 2 diabetes patients. Health Educ. Res. 2013, 28, 539-545. [CrossRef]

45. Li, L.; Yin, X.; Yu, D.; Li, H. Impact of physical activity on glycemic control and insulin resistance: A study of community-dwelling diabetic patients in eastern China. Intern. Med. 2016, 55, 1055-1060. [CrossRef] [PubMed]

46. Nicaise, V.; Crespo, S.; Marshall, S. Agreement between the IPAQ and accelerometer for detecting intervention-related changes in physical activity in a sample of Latina women. J. Phys. Act. Health 2014, 11, 846-852. [CrossRef] 